Itinéraires Itinéraires

Littérature, textes, cultures

\title{
La représentation du corps chez Tatsumi Yoshihiro, maître du manga réaliste (gekiga)
}

\section{Valérie Louison}

\section{(2) OpenEdition}

Journals

Édition électronique

URL : http://journals.openedition.org/itineraires/1571

DOI : 10.4000/itineraires.1571

ISSN : 2427-920X

Éditeur

Pléiade

\section{Édition imprimée}

Date de publication : 1 novembre 2011

Pagination : 173-187

ISBN : 978-2-296-55720-8

ISSN : $2100-1340$

\section{Référence électronique}

Valérie Louison, «La représentation du corps chez Tatsumi Yoshihiro, maître du manga réaliste ( gekiga) », Itinéraires [En ligne], 2011-3 | 2011, mis en ligne le 01 novembre 2011, consulté le 30 avril 2019. URL : http://journals.openedition.org/itineraires/1571; DOI : 10.4000/itineraires.1571

\section{(ब) $(\Theta \Theta$}

Itinéraires est mis à disposition selon les termes de la licence Creative Commons Attribution - Pas d'Utilisation Commerciale - Pas de Modification 4.0 International. 


\title{
La représentation du corps chez Tatsumi Yoshihiro, maître du manga réaliste (gekiga)
}

\begin{abstract}
Tatsumi Yoshihiro is a mangaka whose work is striking through its fundamental humanity. The briefness of these tales is the structure that gives body to the gekiga ("dramatic pictures") style, where Tatsumi excels. The body plays a central role. It is portrayed as much in its collective and social dimension as in its sexual singularity. Sporting their pride, the failure's outfit, the Tatsumian male characters apply themselves to make their personal decline the semantic subject of their lives. The woman is the subject of all the Tatsumian man's torments and all his frustration. Tatsumi's subversive irony gives his graphic short stories a depth of field which makes him an entomologist of the human soul.
\end{abstract}

Keywords : Tatsumi Yoshihiro, manga, body, sex, Japanese society

Mots clés : Tatsumi Yoshihiro, manga, corps, sexe, société japonaise

Tatsumi Yoshihiro est un mangaka dont l'œuvre frappe par sa profonde humanité. La brièveté de ses récits, réelle ou relative, est la structure donnant corps au style du gekiga [« drame dessiné »]. C'est dans l'étroitesse de ce double espace créatif que représentent la case et la bulle, que l'auteur doit réussir à exprimer la dimension à la fois plus réaliste et psychologique de ce nouveau genre de mangas. Cette contrainte d'une narration graphique limitée à 8 ou 16 planches pour les nouvelles les plus brèves et à 24 ou 36 planches pour les plus longues, est une sorte d'étalon esthétique imposé aux artistes de gekiga par le réseau éditorial des librairies de prêt $^{1}$ (kashibon-ya). À cet exercice de style Tatsumi excelle. Une

1. Le réseau des librairies de prêt, en charge de la distribution et de la diffusion de la bande dessinée alternative japonaise, se développe parallèlement à celui officiel des grandes maisons d'édition de presse de Tôkyô, telles que la Kôdansha. La maison d'édition Kôdansha publiait déjà avant-guerre les premiers mensuels de prépublication de manga pour enfant. Seuls les meilleurs titres, ceux qui avaient du succès auprès du public, avaient 
bulle muette, l'intensité dramatique d'une séquence sont autant d'épiphénomènes qui continuent à trouver un écho dans l'inconscient du lecteur et ce bien longtemps après l'énoncé du dénouement.

En avril de l'année 1957, paraît le premier numéro du mensuel de prêt Machi [« La Ville»] auquel Tatsumi collabora en compagnie des futurs grands mangakas de style gekiga tels Saitô Takao, Satô Masaaki, Matsumoto Masahiko ou Ishikawa Fumiyasu. À cette époque l'ensemble de ces auteurs réfléchissent au terme ou à l'expression à donner pour qualifier leur nouveau genre d'histoire dont l'univers narratif très réaliste, comprenant des aspects psychologiques plus complexes, le destine à un public de jeunes adultes et non à celui des enfants. De son côté, Matsumoto trouve le terme de Komaga [« Histoire en vignette »] alors que Tatsumi, aidé dans sa réflexion par son mangaka de frère, forge le néologisme gekiga [« drame dessiné »], insistant sur le contenu et non sur la forme de ce nouveau genre de récits graphiques.

Tatsumi décide d'identifier son nouveau genre de nouvelles graphiques en lui apposant un logo : Gekiga Kôbố2 ["L'Atelier du Gekiga »] à partir du douzième numéro de Machi. Ce logo permet à notre mangaka de distinguer enfin ses œuvres des mangas pour enfant. En janvier 1959, Tatsumi réussira à rallier sous la bannière du gekiga de talentueux artistes, créant ainsi le mouvement graphique du Gekiga Kôbo dont il rédige le manifeste la même année. Manifeste rédigé sur un format de carte postale et que Tatsumi diffuse auprès des plus grands noms du manga dont le maître incontesté : Tezuka Osamu. Le Gekiga Kôbo concevra sous le nom de Skyscraper ${ }^{3}$ sa propre revue de manga. L'inspiration de Tatsumi est de plusieurs ordres. À l'instar d'un écrivain naturaliste du XIX ${ }^{\mathrm{e}}$ français, il effectue un important travail de documentation insufflant une « épaisseur existentielle » à l'œuvre fictionnelle en devenir. Il puise donc dans les rapports de police ou les faits divers relatés dans la presse quotidienne japonaise. Mais au-delà de cette recherche d'effet de réel, Tatsumi, s'inspire également de sa propre existence. Interrogeant les émotions les plus intimes de ses personnages, il montre à son lecteur l'envers (ura) de la société japonaise avec un souci de vraisemblance et de réalité, gage intrinsèque de l'esthétique novatrice de ses nouvelles graphiques. Les thèmes qui y sont abordés sont divers : avortement, suicide, récits de mort et de folie, récits de guerre et de démobilisation, fétichisme et fantasmes sexuels, etc.

droit à une réédition sous forme de livre de poche relié, le format dit tankôbon. Cette production reposait essentiellement sur le genre du story manga pour préadolescent-es et adolescent-es. Le réseau des Kashibon-ya, lui, est fondé sur le principe de l'emprunt en usage dans les bibliothèques, mais à ceci près que la location journalière de manga et autres revues est payante. Le coût modique de cette location, d'environ 2 yens pour 2 jours, fit rapidement du manga de prêt un produit culturel accessible à tous.

2. Tatsumi Yoshihiro, A drifting Life [Une vie à la dérive], Montréal, Drawn \& Quarterly publications, 2009, p. 651.

3. Ibid., p. 730-731. 
Les gekiga de Tatsumi ont donc pour cadre la société contemporaine japonaise, celle des années de sa modernisation (1960-1970). L'auteur y révèle avec un discernement sans égal le fait que la culture japonaise d'après-guerre sait investir tous les modèles de comportements.

L'introduction de valeurs nouvelles dans un Japon post-nucléaire se traduit dans les faits par une réorganisation profonde et une réflexion sur elle-même d'une société en devenir. Ces nombreux changements affectant la société japonaise d'après-guerre, montreront la capacité des individus japonais - comme le souligne Yatabe Kazuhiko dans son article « La société japonaise et la modernité »- « à formuler de nouvelles représentations, à imaginer des récits inédits, à concevoir des mises en scène qui tranchent avec ceux qui ont prévalu jusqu'alors, à proposer de nouvelles pratiques susceptibles de remplacer celles établies... ${ }^{4} \gg$ En un mot, à s'inscrire dans la modernité.

Mais l'adoption des valeurs inhérentes à la modernité n'est pas sans contrepartie. Celles-ci sont sources de tension et d'une angoisse existentielle s'emparant de l'individu qui, au lieu de se sentir intégré à un système de valeurs, s'en trouve exclu pour cause de non-adaptabilité, de « malaise identitaire $»$.

Tatsumi Yoshihiro soulignera par ses récits atypiques les travers de cette modernité en mettant en scène des êtres en rupture de ban, aux corps éprouvés par la vie et s'interrogeant sur ce qu'ils sont réellement.

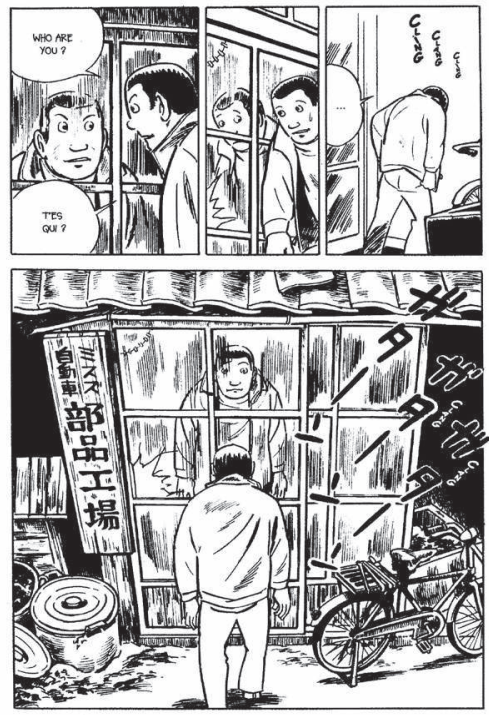

Tatsumi Yoshihiro, planche extraite de « Who are you? Le Scorpion », publiée dans l'album Coup d'éclat, Paris, Vertige Graphic, 2003, p. 94. Sens de lecture japonaise.

4. Yatabe Kazuhiko, «La société japonaise et la modernité », dans Évelyne Douille-Feer (dir.), Japon, le renouveau?, Paris, La Documentation Française, 2002. 
Pour traduire toute la difficulté du dire et du vivre du sujet japonais moderne Tatsumi Yoshihiro fera donc du « fait divers » son principal objet de mise en récit dont la ville est par essence le cadre à la fois narratif et sociétal. C'est au cœur de cet environnement urbain que prennent corps les nouvelles graphiques de Tatsumi. Les agglomérations tokyoïtes et osakiennes, deviennent l'écheveau de ces brefs récits nous restituant le chaos de l'univers tatsumien. Elles sont le théâtre de tranches de vie de personnages en déshérence, ballottés dans l'anonymat des foules et concourent à cet effet de vraisemblance et de réalisme innervant l'œuvre de Tatsumi.

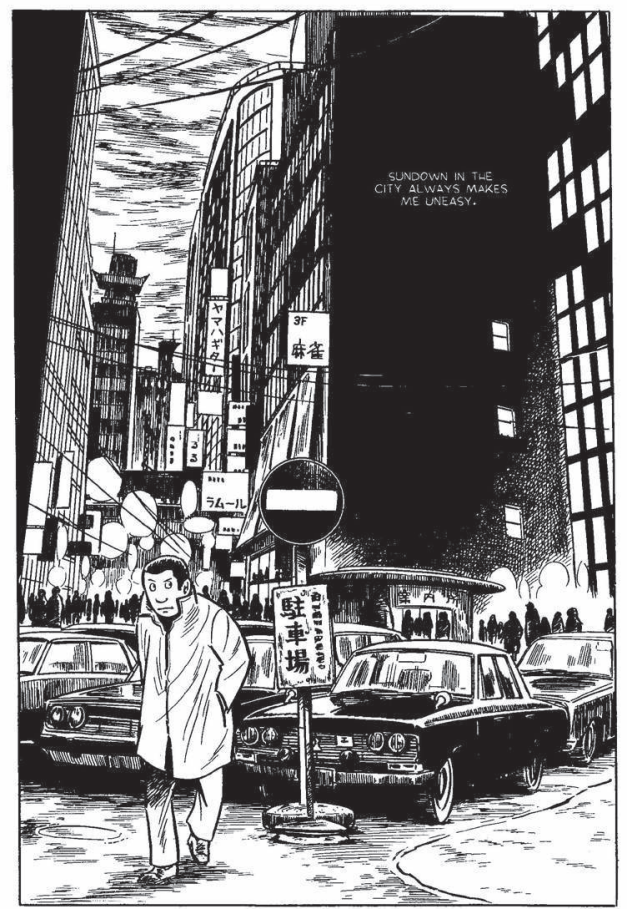

Tatsumi Yoshihiro, planche extraite de « My Hitler» [Mon Hitler], publiée dans le recueil The Push Man, and Other Stories [Le pousseur et autre histoires], Montréal, Drawn \& Quarterly publications, 2005, p. 180.

\section{La déchéance de l'homme tatsumien ou les travers de la modernité}

Tatsumi fait de l'homme ordinaire le personnage clef de ses histoires singulières. Loin de tous les stéréotypes inhérents à la virilité masculine, il brosse des portraits d'hommes à la dérive, traînant leur solitude et leurs turpitudes dans les rues interlopes des cités urbaines. Sur leur front troublé perlent les gouttes de sueur de leur angoisse intérieure. Cabossés par la vie, ils avancent hagards, la tête basse, les épaules voûtées et les poings serrés au creux des poches de leur pardessus d'infortune. Ils avancent dans la foule 
sourde, dans le groupe en ordre de marche dans sa course à la modernité comme un être allant à contre-courant ${ }^{5}$, refusant cet état de chose.

L'homme tatsumien est figuré dans l'expression de sa singularité. Il symbolise en cela l'émergence d'une forme d'individualisme résultant de la modernisation mise en œuvre au cours des années 1960 et 1970 au Japon. Ce corps perturbateur est une représentation métaphorique de l'éclatement du corps social japonais dans l'œuvre de Tatsumi Yoshihiro. Le groupe ne se singularise plus par son esprit de corps marqué par les solidarités villageoises voire familiales mais par les conflits le traversant, et dont l'expression visible est l'émergence de tensions intergénérationnelles. L'ancêtre devient un fardeau de l'existant, fardeau que l'on porte pour mieux en précipiter la fin.

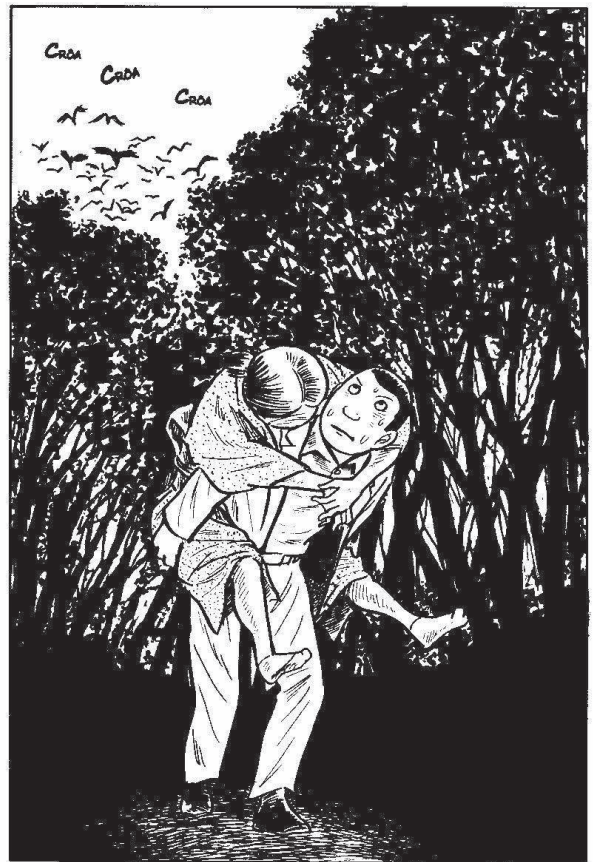

Tatsumi Yoshihiro, planche extraite de « La Colline où abandonner les siens », publiée dans l'album Les Larmes de la Bête, Paris, Vertige Graphic, 2004, p. 30. Sens de lecture japonaise.

Dans sa nouvelle « La colline où abandonner les siens » la mère du jeune Nakamura accomplit cet ultime voyage sur le dos de son fils. Cet acte symbolique obéissant à une coutume « ancestrale » qui veut que toute personne de 70 ans gravisse le sommet d'une montagne pour y mourir s'avère ici perverti à l'extrême. Cette vieille femme accomplira son ultime

5. Tatsumi Yoshihiro, «Who are you? Le Scorpion », publiée dans l'album Coup d'éclat, Paris, Vertige graphique, p. 79, 2003. Sens de lecture japonaise. 
voyage non sur un sommet mais au cœur d'une décharge publique où son corps sans vie, abandonné par un fils qui ne désirait plus avoir à charge cette mère castratrice, pourrira dans un conduit métallique, cercueil improbable pour le cimetière de choses d'une société consumériste.

Le sordide se mêle ici au tragique pour une ballade en dissonance, loin de la poétique Ballade de Narayama [Narayama bushiko] du cinéaste japonais Imamura Shohei.

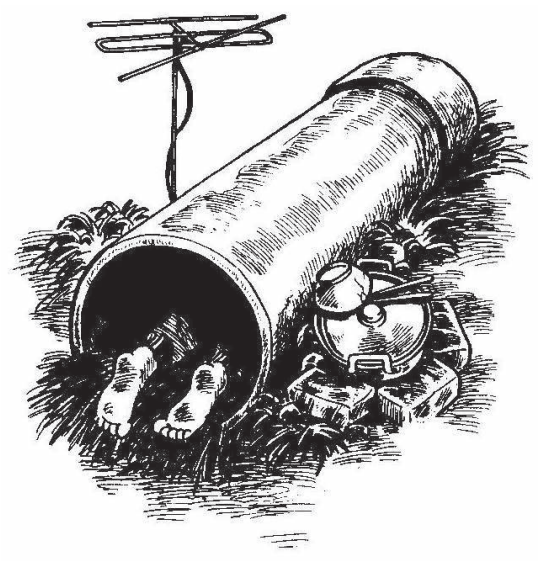

Tatsumi Yoshihiro, vignette extraite de « La Colline où abandonner les siens », publiée dans l'album Les Larmes de la Bête, Paris, Vertige Graphic, 2004, p. 38. Sens de lecture japonaise.

Ce refus du corps vieillissant, des responsabilités familiales symbolise le rejet des traditions d'un temps révolu, rejet dont l'auteur évoque les prémisses sous le règne de l'empereur Hirohito, dans sa nouvelle « L'Enfer ${ }^{6}$ ». Le héros de cette dernière, Koyanagi, se remémore donc ce temps où jeune soldat, attaché à la section militaire d'information, il fut l'un des premiers à photographier la ville d'Hiroshima dévastée. À travers son objectif, il saisissait des scènes irréelles à force d'atrocités : l'ombre d'êtres humains imprimée par l'éclair de la bombe A, dont celle d'un homme penché sur une femme, celle de Mado et Kiyoshi Yamada. Ce « négatif rupestre photographique » surgissant de l'enfer résonnait pour lui comme le dernier témoignage d'amour d'un fils à sa mère. Il apprendra six ans plus tard, par le présumé atomisé Kiyoshi Yamada que ce « négatif rupestre » n'était que le sordide et ironique témoignage du meurtre de sa mère Mado commandité par lui-même auprès d'un de ses amis.

Tatsumi Yoshihiro porte un regard à la fois avisé et périphérique, sur cet Homo japonicus en quête d'un individualisme post-nucléaire. Regard séquentiel et englobant qui s'incarne par la mise en image de l'être japonais dans sa corporalité.

6. Tatsumi Yoshihiro, «L'Enfer» [Jigoku], publiée dans le recueil L'Enfer, Paris, Cornélius, 2008 , p. 13 et p. 22. Sens de lecture japonaise. 


\section{L'homme}

Sacrifiés sur le bûcher du productivisme d'après-guerre, ses personnages masculins sont entravés par une société japonaise névrotique symbolisée par la figure féminine.

Dépossédé de son identité masculine l'homme tatsumien est écartelé entre les reproches d'une mère castratrice et les aspirations matrimoniales d'une petite amie. Cerné par toutes ces attentes affectives, il doit, sous les masques du soldat ou de l'ouvrier, renoncer à son individualité pour mieux devenir un rouage de la société nipponne. En tant que soldat, le voici assigné à inscrire ses actes dans une histoire qui ne lui appartient pas : celle de cette entité abstraite nommée l'Empire nippon. Après-guerre il intègre, sous les traits d'une classe prolétarienne émergente et d'une masse salariale exclusivement masculine, la norme voulant que l'homme soit seul à œuvrer à la modernisation de son pays, à subvenir aux besoins de sa famille et que la femme reste au foyer pour la réussite de cette «magnifique entreprise démocratique $^{7} \gg$.
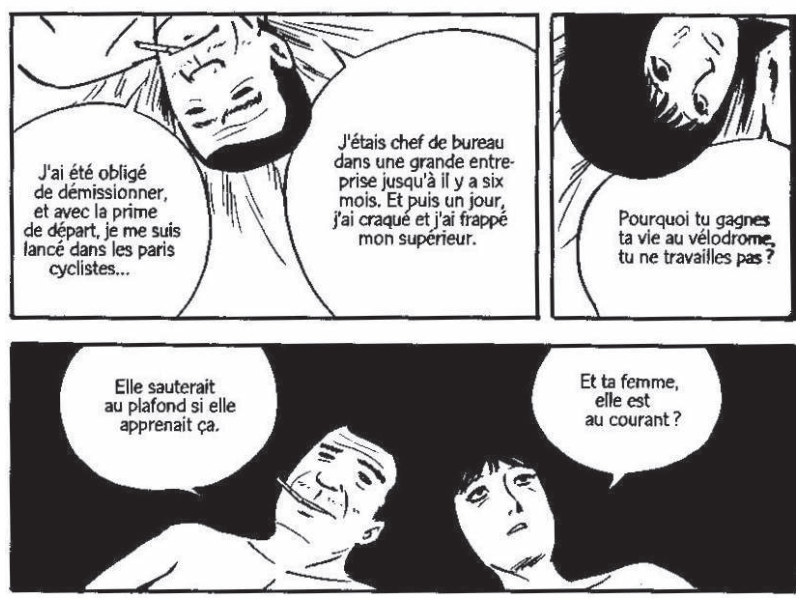

Tatsumi Yoshihiro, vignettes extraites de « Course requiem » [Nenbutsu Race], publiées dans le recueil L'Enfer, Paris, Cornélius, 2008, p. 56-57. Sens de lecture japonaise.

(Première parution dans l'hebdomadaire Manga Times du 24 novembre 1973.)

Frustrés par une vie n'ayant aucune raison d'être, certains de ces personnages masculins iront jusqu'à assassiner l'objet de leur frustration incarné par le personnage féminin. Les plus faibles d'entre eux « révèlent leurs faiblesses à des inconnues, pour rester dignes devant leur femme ${ }^{8}$ ", comme c'est le cas pour le personnage principal de la nouvelle « La Course à la prière au Bouddha » traduite également sous le titre de « La Course requiem », ou deviennent sous les traits d'artistes ratés ou de gigolos capricieux, de vulgaires animaux de compagnie, pour des femmes nourricières

7. Muriel Jolivet, Homo Japonicus, Arles, Éd. Philippe Picquier, 2002, p. 182.

8. Ibid., p. 138. 
et volages ${ }^{9}$. L'homme devient alors l'objet d'un rapport de force inversé : n'étant plus que l'ombre d'un chien, le voici à la merci d'une femme souveraine et maîtresse, disposant à loisir de ce corps masculin.

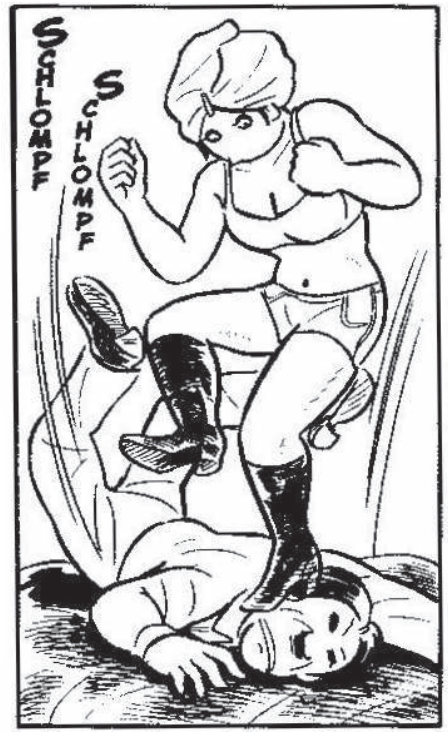

Tatsumi Yoshihiro, vignette extraite de « Le Pied», publiée dans l'album Les Larmes de la Bête, Paris, Vertige Graphic, 2004, p. 76.

Dans certains de ces récits, Tatsumi peut encore faire preuve d'un plus grand cynisme dans la représentation de la déchéance existentielle de ses personnages masculins. Pour ce faire, il leur fait endosser les guenilles de SDF.

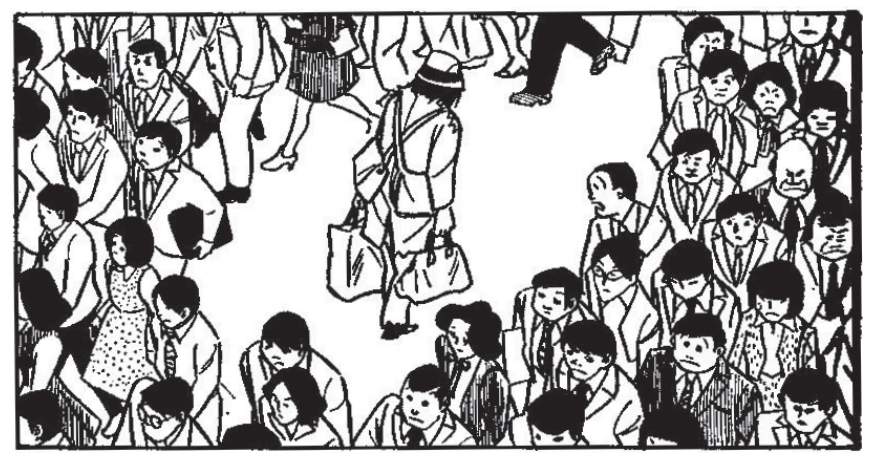

Tatsumi Yoshihiro, vignette extraite de « Hôtel du métro » [Chikatetsu Hotel], publiée dans le recueil L'Enfer, Paris, Cornélius, 2008, p. 73. Sens de lecture japonaise. (Première parution dans Manga Goraku du 10 avril 1975. )

9. À ce sujet, se référer à « La nourrice » et « Une ville dans le creux de la main », deux nouvelles graphiques de Tatsumi Yoshihiro publiées respectivement en mai 1970 et décembre 1973. 
Abandonnés par leurs proches, oubliés de tous, ces parias des temps modernes sont les icônes des exclus d'un système laissant sur le quai les laissés-pour-compte du train du « miracle japonais ». Certains sont même éjectés de ce «train en marche », tristes victimes collatérales des soubresauts des crises économiques conjoncturelles du pays. Dans la nouvelle intitulée « Télescope » (1969), Tatsumi nous narre à la première personne l'histoire d'un homme marginalisé suite à un accident de voiture qui lui a causé à la fois la perte de sa jambe et de son travail. Disposant à loisir de tout le temps de l'errance, notre protagoniste l'emploie à s'introduire, via les télescopes de la terrasse panoramique d'un grand magasin nippon, dans l'intimité des appartements situés juste en face.

Tatsumi met également en lumière le rapport de l'individu à la société par le biais du fait divers. Cette irruption du genre du fait divers au sein de la fiction qui est en train de se jouer permet à l'auteur de réaliser un télescopage volontaire entre réalité potentielle et fiction. Ainsi ce mélange des genres intervient à un moment où la tension dramatique est à son paroxysme. C'est l'instant où l'un des protagonistes du récit commet un acte irréversible le condamnant à la prison de son enfer intérieur et à son exclusion sociale.

Ce temps du paroxysme, qui n'est autre que l'expression cathartique du côté obscur des personnages, est contenu non pas dans l'objet figuré, en l'occurrence un meurtre, un viol ou un infanticide, mais dans l'esthétique de mise en abîme graphique à laquelle a recours l'auteur pour nous le représenter. Pour ce faire Tatsumi emploie un dispositif clef : la case dite « en négatif» que l'on pourrait également nommer : dispositif de la case « en ombre chinoise».

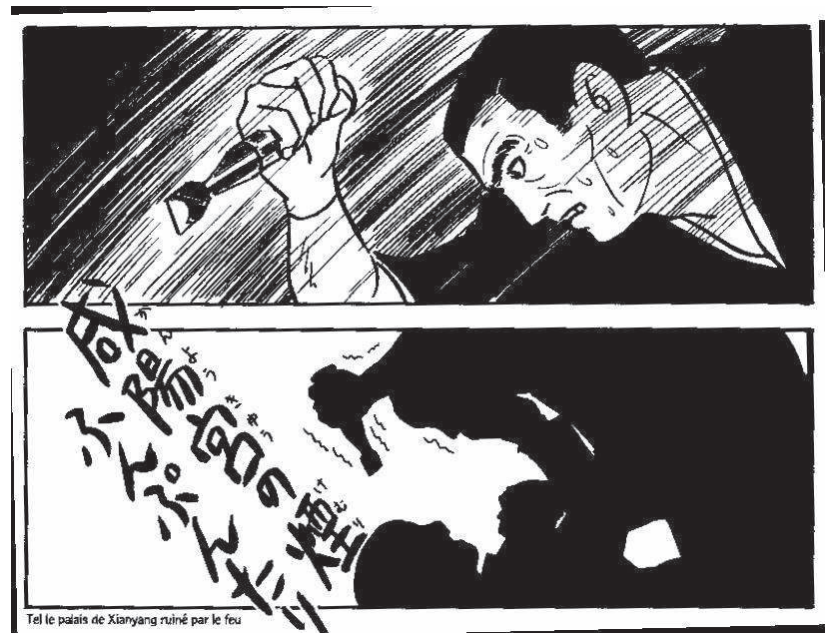

Tatsumi Yoshihiro, vignettes extraites de « Le Théâtre de la vie » [Iro Zange], publiées dans le recueil L'Enfer, Paris, Cornélius, 2008, p. 221. Sens de lecture japonaise. (Première parution dans le supplément du Young Comic du 8 janvier 1974.) 
Le dispositif de cette case en négatif représente un arrêt sur image du récit en cours et concentre par ce jeu en négatif la tension de l'événement à venir ou celui qui est en train de se dérouler, sous nos yeux. Cette case symbolise donc la chute irréversible des personnages dans le puits sans fond de leurs échecs et de leurs névroses. Le corps, bien que représenté en négatif, y joue un rôle central.

En usant ainsi de ce dispositif, Tatsumi élabore une signature graphique directement empruntée de l'esthétique cinématographique en usage dans les films à sujets contemporains du début des années 1960, nommés gendaigeki et dont le chef de file incontesté fut Kurosawa Akira, mais également de celle du film noir américain et européen des années 1950-1960. Grâce à ces influences largement revendiquées, il sut élaborer et développer un style bédéique marqué par une volonté d'austérité et du refus d'une représentation banalisée du spectaculaire.

Ce temps du passage à l'acte met en question le rapport de l'individu à la société japonaise qui est en train de se moderniser. Il agit comme un révélateur des défaillances d'une société envers les hommes qui contribuent corps et âme à son nouvel essor économique et culturel et à son rayonnement international. Il agit comme le signe ${ }^{10}$ de la difficulté d'être au monde et de vivre au rythme des cadences effrénées ${ }^{11}$ de la course à la productivité engagée après-guerre.

Les personnages masculins tatsumiens sont donc dotés d'une psyché ambiguë dont la femme est le principal sujet des tourments et des frustrations.
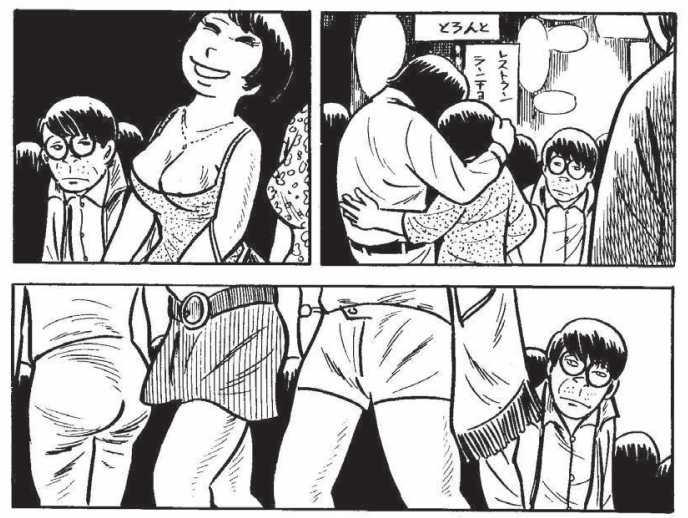

Tatsumi Yoshihiro, vignettes extraites de « Night falls again » [La nuit tombe à nouveau], publiées dans le recueil Good-Bye, Montréal, Drawn \& Quarterly, 2008, p. 149.

10. Maurice Merleau Ponty, « Sur les faits divers », dans Signes, Paris, Gallimard, 1954, p. 389-391.

11. Tatsumi Yoshihiro, « Monkey mon amour », publiée dans l'album Good Bye, Paris, Vertige Graphic, 2005, p. 47. Sens de lecture japonaise. 
Portant le masque d'un piranha ou d'un Hitler qui accable le « héros » de tous les maux, la femme se fait également nourricière et protectrice. Mais avant tout elle reste, pour ces hommes en souffrance, cet objet de désir au puissant pouvoir érotique. Objet de désir pour lequel ils sont prêts à laisser libre cours à leur libido perverse empreinte de fantasmes inavouables et de déviances fétichistes.
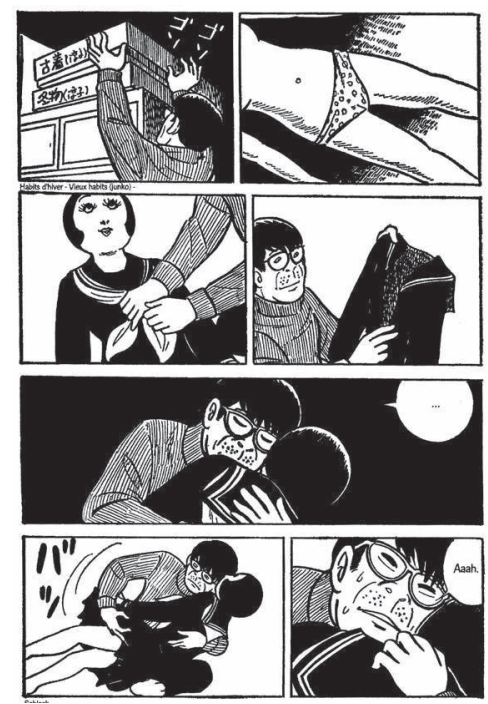

Tatsumi Yoshihiro, planche extraite de «Une fille dans la poche » [Pocketo no naka no onna], publiée dans le recueil L'Enfer, Paris, Cornélius, 2008, p. 145. Sens de lecture japonaise.

(Première parution dans le supplément du Young Comic du 9 janvier 1973.)

Objet de désir dont la réelle possession ne s'incarne que dans le viol, la mort ou le cannibalisme : dans « La grue de papier », un long récit en image de 19 planches publié en octobre 1984, Tatsumi interpelle l'auteur d'un des faits divers les plus énigmatiques et terrifiants de la seconde moitié $\mathrm{du} \mathrm{Xx}^{\mathrm{e}}$ siècle, Sagawa Issei, « le Japonais cannibale».

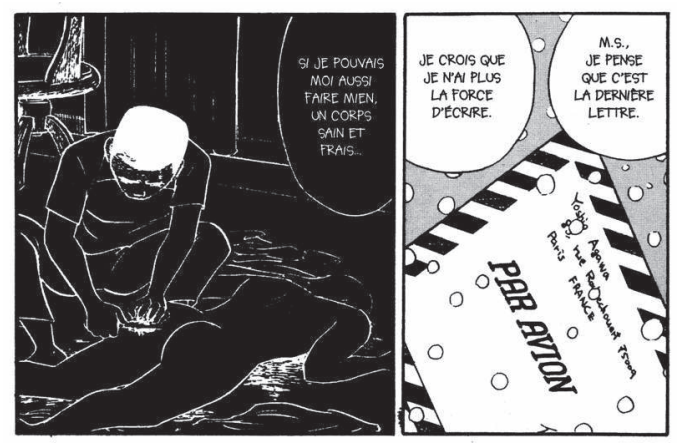

Tatsumi Yoshihiro, vignettes extraites de « La Grue de papier. Lettre à M.S. », publiées dans l'album Good Bye, Paris, Vertige Graphic, 2005, p. 26. Sens de lecture japonaise. 


\section{La femme}

Tatsumi affectionne particulièrement les personnages féminins asservis, symbole fétichiste d'une société nippone aveugle face à son néo-conformisme pervers. Cependant, au-delà de leur avilissement physique et moral, ces anges ivres, pour lesquels aucun espoir de rédemption n'est annoncé, officient en figures réprobatrices d'une société cruelle pour les indigents et autres cabossés de la vie.

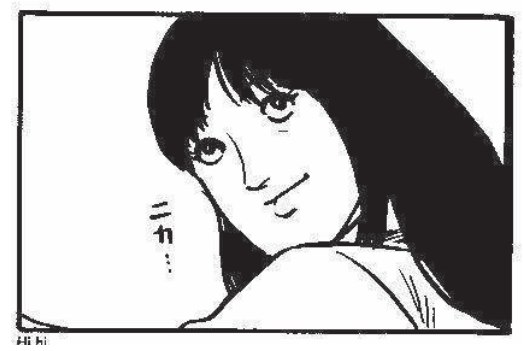

Tatsumi Yoshihiro, vignette extraite de «Course requiem » [Nenbutsu Race], publiée dans le recueil L'Enfer, Paris, Cornélius, 2008, p. 49. Sens de lecture japonaise.

(Première parution dans l'hebdomadaire Manga Times du 24 novembre 1973.)

Une jeune femme aux traits réguliers, à l'air ingénu, à la chevelure noir corbeau, tombant délicatement sur les épaules, apparaît comme un des personnages fétiches de Tatsumi. Adepte de l'avortement ou prostituée occasionnelle, comme dans son récit intitulé «L'amour de la futur mariée » [Ai no hanayome], cette beauté hautaine s'avère être l'archétype féminin récurrent dans l'œuvre du mangaka.

Sa destinée est liée à la mort, elle est le symbole des dénouements tragiques de ces brefs récits empreints de l'univers de la rubrique des faits divers urbains des quotidiens tokyoïtes.

Cette femme apparaît à la fois comme le réceptacle vivant de la mémoire japonaise et comme celui de son devenir. À l'image de l'hôtesse de bar racontant l'histoire du Japon du cinéaste Imamura Shohei, les prostituées des bas-fonds des cités japonaises qui peuplent les histoires de Tatsumi battent en brèche le consensus du silence entourant les événements d'Hiroshima et de Nagasaki. Ce silence apparaît dans un Japon d'aprèsguerre en proie à la honte de la défaite (haisen) comme un refuge salvateur, comme le « voile réparateur du vaincu ». Elles, les fleurs de macadam, les "Pan Pan », s'octroient le droit à la parole, celle du désespoir sans fard de leur existence dans ce Japon en manque de repère et en perte d'identité.

La nouvelle graphique « Good Bye » représente symboliquement ce « point d'origine » (kigen) qu'est la défaite. La destruction des liens identitaires entre les êtres atteint ici son paroxysme dans la figuration elliptique d'un coït incestueux entre un soldat japonais démobilisé et sa fille Mariko vivant du commerce de ses charmes auprès des soldats de l'armée d'occupation. 

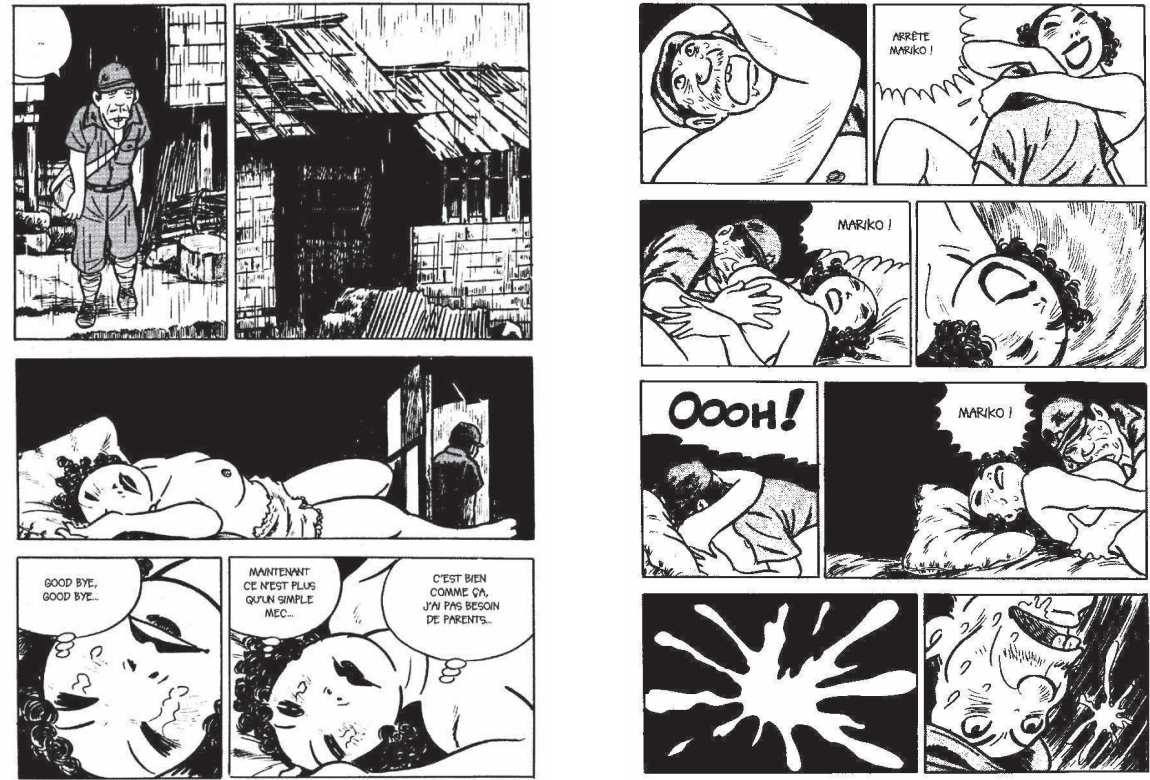

Tatsumi Yoshihiro, vignettes extraites de " Night falls again » [La nuit tombe à nouveau], publiées dans le recueil Good-Bye, Montréal, Drawn \& Quarterly, 2008, p. 148.

Le personnage féminin pour Tatsumi n'est pas là pour protéger la libido des hommes mais pour mettre en évidence leurs travers, leurs déviances. « Cet obscur objet du désir » ne reste pas à sa place, c'est-à-dire désincarné et surtout muet. Il dit à qui veut - et peut - l'entendre toute cette souffrance subie au nom de l'Empire, au nom du père, au nom de la famille.

La parole de femme se libère de cette "sainte trinité » et exhume de la mémoire collective les heures sombres de ce Japon fier et belliqueux de la Seconde Guerre mondiale.

Ainsi c'est par le biais des souvenirs fictifs des prostituées Ozumi et Kikumaru, héroïnes du « Journal de guerre d'une prostituée ${ }^{12}$ », mais ô combien empreints de faits historiques, que nous est révélée une des pages les plus sombres et abjectes de l'histoire de cette armée impériale. Ce récit bouleversant évoque le triste sort réservé à des milliers de femmes asiatiques lors de la Seconde Guerre mondiale : nombre d'entre elles ont été enrôlées par l'armée japonaise de gré, grâce à de vils subterfuges tel la promesse d'embauche, ou de force. Elles ont alors été contraintes de se prostituer dans « leurs bordels militaires ambulants ${ }^{13} »$.

12. Tatsumi Yoshihiro, "Journal de guerre d'une prostituée » [Shôfu no senki], publiée dans le recueil L'Enfer, Paris, Cornélius, 2008, p. 284. Sens de lecture japonaise (première parution dans l'hebdomadaire Manga Times du 29 septembre 1978).

13. George Hicks, Les Esclaves sexuelles de l'armée japonaise, traduit de l'anglais par Françoise Thévenod, Paris, Éd. Jacques Grancher, 1996. 
Ces femmes dites de réconfort sont principalement coréennes, mais aussi taïwanaises, indonésiennes et japonaises. Leur asservissement sexuel devait permettre de maintenir le moral des troupes nippones et pour les femmes de réconfort qui sont de nationalité japonaise, concourir tout « naturellement» à l'effort de guerre. Au même titre que les soldats, ces femmes doivent se dévouer corps et âme à l'empereur et combattre pour la victoire du Japon. Toute action menée en ces temps de guerre par un sujet japonais est, et demeure, patriotique. Tout cela était pour le bien du pays ${ }^{14}$ !

Cette prise de parole par les personnages féminins, à l'encontre de l'État-nation japonais, est aussi la marque d'une occidentalisation en marche.

Au-delà de la figure de la prostituée, Tatsumi nous livre par sa narration et ses cadrages incisifs, des portraits réalistes de femmes modernes japonaises, secrétaire, employée de bureau. Nouvellement émancipées, ces femmes, si singulières dans leur apparente banalité, demeurent pourtant soumises aux diktats des conventions sociales japonaises.
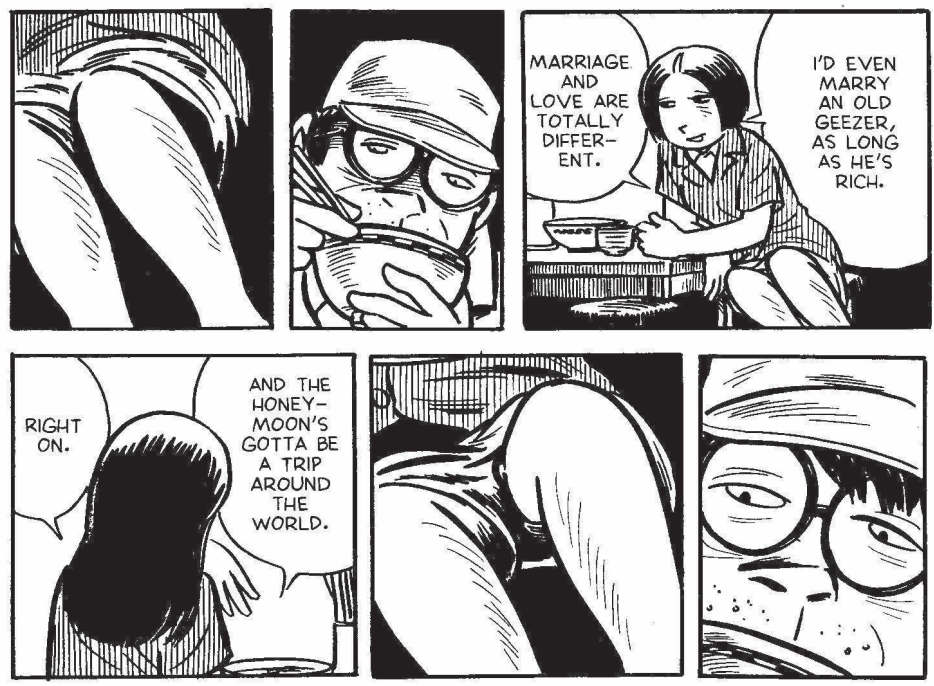

Tatsumi Yoshihiro, planches extraites de « Good Bye »,

dans l'album Good Bye, Paris, Vertige Graphic 2005, p. 72-73. Sens de lecture japonaise.

Le mariage semble le seul moyen pour espérer obtenir la sécurité matérielle et sociale. La limite des récits de Tatsumi est de ne proposer aucune issue positive pour ses héroïnes. Elles restent inexorablement tributaires du pouvoir des hommes, incapables de prendre leurs destinées en main et de s'affranchir du poids de la société moderne.

14. Tatsumi Yoshihiro, «Journal de guerre d'une prostituée » [Shôfu no senki], publiée dans le recueil L'Enfer, Paris, Cornélius, 2008, p. 290. Sens de lecture japonaise (première parution dans l'hebdomadaire Manga Times du 29 septembre 1978). 


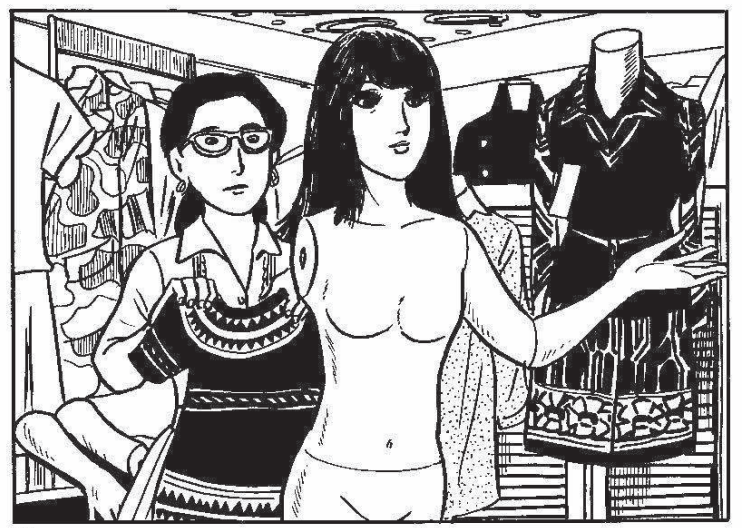

Tatsumi Yoshihiro, vignette extraite de « Une ville dans le creux de la main » [Tenohira no Machi], publié dans le recueil L'Enfer, Paris, Cornélius, 2008, p. 116. Sens de lecture japonaise. (Première parution dans l'hebdomadaire Manga Times du 22 décembre 1973.)

Ainsi, le corps est ce qui réduit à sa plus simple expression le personnage féminin tatsumien. Violenté, meurtri, drogué et abusé à son insu, ce corps féminin qui est à la merci du bistouri des faiseurs d'ange des temps modernes est aussi par essence le catalyseur des pulsions sexuelles et autres pratiques fétichistes des hommes.

«Femme objet », dépossédée de son corps et à la fois consciente de sa beauté ${ }^{15}$, la femme est dans l'œuvre de Tatsumi le vecteur du changement, le personnage par qui le drame arrive. Tatsumi parvient à capter l'intensité psychologique de ses personnages et à créer un rythme et une impulsion qui se révèlent à la lecture de chacune de ses œuvres. Dans la vérité qu'elles suggèrent, un grand nombre d'entre elles sont sans concession, saisissant l'essence même de ces êtres aux regards inquiets et aux visages qui suintent l'angoisse. Accablés par leur phobie sociale, les personnages tatsumiens sont prisonniers de leurs errances intérieures. Cependant ils intègrent une ironie consciente, s'avérant des plus déconcertantes pour tout lecteur.

Ainsi l'ironie subversive de Tatsumi Yoshihiro donne à ses nouvelles graphiques une profondeur de champ qui en fait un entomologiste de l'âme humaine où les corps, humains et urbains, individuels et collectifs, sont représentés dans leur plus simple humanité.

Valérie Louison Bibliothèque nationale de France

15. Dans la nouvelle de Tatsumi intitulée « Un long, très long été » [ Nagai nagai natsu ], publiée en 1971, Maki est une jeune femme qui ne supporte pas l'idée de voir la beauté de son corps, seule chose dont elle est fière, mise à mal par les déformations d'une grossesse. Elle avorte sans le consentement de Hara Jun, son petit ami, puis met fin à ses jours. 